\title{
À propos des prescriptions hors-AMM
}

\author{
Pascale Jolliet \\ Service de Pharmacologie Médicale, Faculté de Médecine, Nantes, France
}

Le médecin, après avoir établi un diagnostic, doit prescrire le traitement le plus adapté à son patient, celui qui offrira le meilleur rapport bénéfice/risque dans une situation donnée. Tout médicament préparé industriellement doit être pourvu d'une autorisation de mise sur le marché (AMM), nationale ou communautaire selon l'article L.5121-8 du code de la santé publique. Le dossier fourni par l'industriel à l'Agence française de sécurité sanitaire des produits de santé (Afssaps) ou à l'European Medicines Agency (EMA) regroupe la totalité des informations et études permettant d'évaluer le bien-fondé scientifique de l'autorisation et permettra d'évaluer 3 critères essentiels : la qualité, l'efficacité et la sécurité. L'AMM garantit un rapport bénéfice/risque acceptable pour la (ou les) indication(s) retenue(s) et précise dans le résumé des caractéristiques du produit (RCP) les modalités d'administration et de suivi optimales. Elle constitue le référentiel de bon usage du médicament. Ainsi tout médecin au quotidien a la chance de pouvoir se reposer sur des guides solides et sécurisants, validés par un travail d'expert reconnu, que constituent les RCP, la stratégie thérapeutique des avis de la Commission de Transparence ainsi que les recommandations de bonne pratique de la Haute Autorité de Santé (HAS) et de l'Afssaps. La prescription d'un médicament dans le cadre de l'AMM est donc la règle.

La prescription de médicaments hors-AMM peut se faire dans deux cas expressément spécifiés par la Loi et les règlements : les autorisations temporaires d'utilisation (ATU) nominative ou de cohorte délivrées par l'Afssaps, et la recherche biomédicale. Mais la prescription hors-AMM n'est par ailleurs interdite par aucun texte. Aussi tout médecin, notamment à l'hôpital, est autorisé à prescrire un médicament hors-AMM conformément aux règles déontologiques : les articles R-4127-8, R-4127-40 et R-4127-32 du code de la santé publique disposent que «dans les limites fixées par la loi, le médecin est libre de ses prescriptions qui seront celles qu'il estime les plus appropriées en la circonstance» mais aussi que «le médecin doit s'interdire, dans les investigations et interventions qu'il pratique comme dans les thérapeutiques qu'il prescrit, de faire courir au patient un risque injustifié » et qu'il doit s'appuyer «sur les données acquises de la science ». Les prescriptions hors-AMM sont notamment très fréquentes en cancérologie et en néonatalogie.

Sur le plan juridique, la prescription d'un médicament hors-AMM doit répondre aux règles du droit de la responsabilité médicale ; ainsi la responsabilité civile ou pénale du médecin peut être engagée. On comprend l'indispensable nécessité d'une délivrance d'information complète et précise au patient, y compris en ce qui concerne les conditions de remboursement par les régimes obligatoires d'assurance maladie. Le caractère non remboursable doit de plus figurer sur l'ordonnance.

L'évolution des connaissances scientifiques est rapide, les AMM ne sont pas toujours actualisées (délais administratifs, choix du laboratoire pharmaceutique... ). La prescription d'un médicament « hors-AMM » est parfois nécessaire pour le patient; il est alors préférable qu'elle soit choisie de façon collégiale et effectuée dans un cadre très spécialisé garantissant la qualité du suivi du patient, sa bonne information, mais aussi la vérification a posteriori que la prescription était bien indiquée ainsi que l'évaluation des pratiques professionnelles. 